\title{
Co-located quantitative trait loci mediate resistance to Agrobacterium tumefaciens, Phytophthora cinnamomi, and $P$. pini in Juglans microcarpa $\times J$. regia hybrids
}

\author{
Ramesh K. Ramasamy ${ }^{1}$, Ming-Cheng Luo (1)', Charles A. Leslie', Dianne Velasco ${ }^{1}$, Natalia Ott², Ali McClean²,

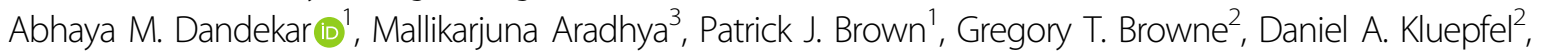 \\ Andreas Westphal ${ }^{4}$ and Jan Dvorak (iD ${ }^{1}$
}

\begin{abstract}
Soil-borne plant pathogens represent a serious threat that undermines commercial walnut (Juglans regia) production worldwide. Crown gall, caused by Agrobacterium tumefaciens, and Phytophthora root and crown rots, caused by various Phytophthora spp., are among the most devastating walnut soil-borne diseases. A recognized strategy to combat soil-borne diseases is adoption of resistant rootstocks. Here, resistance to A. tumefaciens, $P$. cinnamomi, and $P$. pini is mapped in the genome of Juglans microcarpa, a North American wild relative of cultivated walnut. Half-sib $J$. microcarpa mother trees DJUG 31.01 and DJUG 31.09 were crossed with J. regia cV. Serr, producing 353 and 400 hybrids, respectively. Clonally propagated hybrids were genotyped by sequencing to construct genetic maps for the two populations and challenged with the three pathogens. Resistance to each of the three pathogens was mapped as a major QTL on the long arm of J. microcarpa chromosome 4D and was associated with the same haplotype, designated as haplotype $b$, raising the possibility that the two mother trees were heterozygous for a single Mendelian gene conferring resistance to all three pathogens. The deployment of this haplotype in rootstock breeding will facilitate breeding of a walnut rootstock resistant to both crown gall and Phytophthora root and crown rots.
\end{abstract}

\section{Introduction}

Persian (English) walnut (Juglans regia) is an important nut tree crop worldwide. China, Iran, and the United States lead in world production of commercial walnuts (FAOSTAT), while California produces virtually all of the US crop. Commercial walnut orchards often incur serious losses from soil-borne pathogens, primarily Agrobacterium tumefaciens (causal agent of crown gall), numerous Phytophthora spp. (Phytophthora root and crown rots), Armillaria mellea (Armillaria root rot), and phytopathogenic

\footnotetext{
Correspondence: Jan Dvorak (jdvorak@ucdavis.edu)

'Department of Plant Sciences, University of California, Davis, USA

USDA-ARS Crops Pathology and Genetics Research Unit, Department of Plant Pathology, University of California, Davis, USA

Full list of author information is available at the end of the article
}

nematode species (root lesion, root-knot, and ring nematodes).

The strategy of cultivating scion varieties on diseaseresistant rootstocks, usually interspecific hybrids or wild relatives, has been effective in managing soil-borne diseases. About $80 \%$ of commercially grown walnuts in California are grafted onto Paradox rootstocks, which are hybrids derived from the locally adapted Northern California black walnut, J. hindsii, pollinated with J. regia pollen ${ }^{1}$. Additional, commercially available, hybrid rootstocks include 'RX1' (a clonally propagated J. microcarpa $\times J$. regia hybrid), selected for resistance to Phytophthora spp. ${ }^{2}$, and 'VX211' (a J. hindsii $\times$ J. regia hybrid), tolerant to lesion nematode (Pratylenchus vulnus).

\section{(c) The Author(s) 2021}

(c) Open Access This article is licensed under a Creative Commons Attribution 4.0 International License, which permits use, sharing, adaptation, distribution and reproduction in any medium or format, as long as you give appropriate credit to the original author(s) and the source, provide a link to the Creative Commons license, and indicate if changes were made. The images or other third party material in this article are included in the article's Creative Commons license, unless indicated otherwise in a credit line to the material. If material is not included in the article's Creative Commons license and your intended use is not permitted by statutory regulation or exceeds the permitted use, you will need to obtain permission directly from the copyright holder. To view a copy of this license, visit http://creativecommons.org/licenses/by/4.0/. 
Screening a collection of hybrids of diverse species of Juglans with J. regia revealed resistance to both $A$. tumefaciens and Phytophthora spp. in J. microcarpa ${ }^{2,3}$, a small statured tree native to dry regions of Texas, New Mexico, Oklahoma, and Kansas. Juglans microcarpa and J. regia diverged about 8 million years ago ${ }^{4}$, but they readily hybridize. The hybrids are fully male sterile and $90-99 \%$ female sterile. Both species have $n=16$ chromosomes, which originated by a whole-genome duplication of an ancestral genome with $n=8$ chromosomes ${ }^{5}$.

Resistance to A. tumefaciens has been observed in a number of woody perennials, such as Vitis spp., ${ }^{6,7}$, Prunus spp. ${ }^{8}$, Malus spp. ${ }^{9,10}$, and Rosa spp. ${ }^{11}$, in addition to Juglans spp. ${ }^{3}$, as well as in herbaceous plants, such as Pisum sativum ${ }^{12}$ and Arabidopsis thaliana ${ }^{13}$. In A. thaliana, resistance to $A$. tumefaciens was shown to be due to a deficiency in T-DNA integration ${ }^{13,14}$. Resistance to Phytophthora cinnamomi in woody perennials was investigated in Japanese and Chinese chestnuts (Castanea crenata and C. mollissima, respectively), where it was mediated by two major quantitative trait loci (QTLs) and several minor QTLs ${ }^{15,16}$. The molecular mechanism of this resistance has not been determined.

QTL mapping relies on the detection of association between a phenotypic trait and genetic markers ${ }^{17}$. Single nucleotide polymorphisms (SNPs) are currently nearly universally used as genetic markers. SNPs have been discovered and characterized in the J. regia genome ${ }^{18-20}$. Due to the divergence of the J. microcarpa and J. regia genomes, J. regia SNP markers are not useful for mapping the J. microcarpa genome. The genotyping-by-sequencing (GBS) approach ${ }^{21}$ is a viable strategy for developing new SNP markers for genotyping $J$. microcarpa $\times J$. regia hybrids and the construction of maps of both genomes.

This paper describes the development of two populations of Juglans hybrids created by crossing J. microcarpa half-sib mother trees DJUG 31.01 (henceforth 31.01) and DJUG 31.09 (henceforth 31.09) with the cultivated J. regia cv. Serr. These populations were genotyped by GBS, and genetic maps of the parental genomes were constructed. Segregation for resistance to A. tumefaciens, $P$. cinnamomi, and $P$. pini among the hybrids was analyzed in each mapping population (MP), and resistance was mapped as
QTLs. An assembled and annotated J. microcarpa genome sequence $^{4}$ was used to analyze the QTLs.

\section{Results}

GBS marker development and linkage map construction

The $F_{1}$ hybrids $31.01 \times c v$. Serr and $31.09 \times c v$. Serr and their parents produced a total of $1.9 \times 10^{10}$ useful reads, $\approx 2.3 \times 10^{6}$ per hybrid. Low-quality SNPs as defined in Materials and methods and SNPs with more than 40\% missing data were removed. Remaining missing SNPs were imputed and wrong allele calls were corrected with the FSFHap algorithm implemented in Tassel $v 5^{22}$, which uses parental haplotypes to impute SNPs of progeny. SNPs showing segregation distortion were also removed. A database of SNPs used in linkage map construction including $200 \mathrm{bp}$ of surrounding sequence was created (Supplementary Table 1).

A plot of the first two principal component analysis (PCA) axes revealed the identity of hybrid progenies. Each parent formed a distinct cluster, and hybrids formed two additional clusters (Supplementary Fig. 1). Five and two individuals among the hybrids clustered with J. microcarpa and cv. Serr, respectively, rather than with the hybrids, and were excluded since their identity was questionable.

A total of 353 hybrids in the $31.01 \times \mathrm{cv}$. Serr population and 400 hybrids in the $31.09 \times \mathrm{cv}$. Serr population were used in linkage analyses (Table 1, Supplementary Table 2). Segregation of the markers in the two MPs was used to construct linkage maps for the J. microcarpa $(\mathrm{Jm})$ and $J$. regia (Jr) genomes. Each map consisted of 32 linkage groups (LGs), 16 for each parental genome (Supplementary Table 3 and Supplementary Fig. 2).

The linkage map of the $J m$ genome constructed from the $31.01 \times \mathrm{cv}$. Serr MP consisted of 998 SNP markers, which were in 882 co-segregating marker bins, while the linkage map of the $J r$ genome consisted of 807 SNP markers, which were in 672 co-segregating marker bins (Table 1). The total length of the $J m$ genome was $984.6 \mathrm{cM}$ with individual LGs ranging from 26.1 to $96.3 \mathrm{cM}$ and a mean of $61.5 \mathrm{cM}$ (Table 1). The SNP markers adequately covered the linkage groups with a sole exception of LG Jm8S which had a $40 \mathrm{cM}$ gap on the long arm (Supplementary Fig. 2).

Table 1 Total numbers of GBS SNP markers, the numbers co-segregating marker bins, and the total lengths of linkage maps developed from the J. microcarpa $31.01 \times \mathrm{cv}$. Serr and J. microcarpa $31.09 \times \mathrm{Cv}$. Serr mapping populations

\begin{tabular}{lllllll}
\hline Mapping population & Genome & Markers (no.) & Marker bins (no) & Total length (cM) & Average LG length (cM) & LG length range (cM) \\
\hline $31.01 \times$ cv. Serr & Jm & 998 & 882 & 984.6 & 61.5 & $26.1-96.3$ \\
& Jr & 807 & 672 & 1326.0 & 82.9 & $50.1-125.2$ \\
$31.09 \times$ cv. Serr & $J m$ & 950 & 619 & 1115.7 & 69.7 & $50.8-101.0$ \\
& Jr & 748 & 625 & 1357.4 & 84.8 & $53.3-132.3$ \\
\hline
\end{tabular}


Table 2 Percentages of crown length rotted (PCLR) and percentages of root length rotted (PRLR) of the clones of the $F_{1}$ J. microcarpa $31.01 \times \mathrm{cv}$. Serr and J. microcarpa $31.09 \times \mathrm{cv}$. Serr hybrids grown in un-inoculated, control soil and clones grown in soil inoculated with $P$. cinnamomi or $P$. pini

\begin{tabular}{llllllll}
\hline Pathogen & Population & Hybrids (no.) & $\begin{array}{l}\text { Clones phenotyped per } \\
\text { hybrid (no.) }\end{array}$ & $\begin{array}{l}\text { PCLR control } \\
\text { soil }^{\text {a }} \text { (\%) }\end{array}$ & $\begin{array}{l}\text { PCLR inoculated } \\
\text { soil }^{\mathbf{a}} \text { (\%) }\end{array}$ & $\begin{array}{l}\text { PRLR control } \\
\text { soil }^{\mathbf{a}}\end{array}$ & $\begin{array}{l}\text { PRLR inoculated } \\
\text { soil }^{\mathbf{a}}(\%)\end{array}$ \\
\hline P. cinn. & $31.01 \times$ cv. Serr & 249 & 10.2 & $1.4 \mathrm{a}$ & $33.2 \mathrm{a}$ & $22.5 \mathrm{a}$ & $44.6 \mathrm{a}$ \\
& $31.09 \times$ cv. Serr & 223 & 11.3 & $2.3 \mathrm{a}$ & $24.2 \mathrm{~b}$ & $15.5 \mathrm{~b}$ & $34.6 \mathrm{~b}$ \\
P. pini & $31.01 \times$ cv. Serr & 246 & 11.5 & $1.2 \mathrm{a}$ & $36.5 \mathrm{a}$ & $20.5 \mathrm{a}$ & $36.2 \mathrm{a}$ \\
& $31.09 \times$ cv. Serr 275 & 11.5 & $2.2 \mathrm{a}$ & $22.8 \mathrm{~b}$ & $14.2 \mathrm{~b}$ & $27.4 \mathrm{~b}$ \\
\hline
\end{tabular}

a Values in columns for the same pathogen followed by the same letter do not differ at the $P<0.001$ (t-test) significance level

The total length of the $J r$ genome, $1326.0 \mathrm{cM}$ (Table 1$)$, was significantly longer than that of the $J m$ genome $(P=$ 0.0005 , paired $t$-test) with individual LG ranging from 50.1 to $125.2 \mathrm{cM}$ and a mean of $82.9 \mathrm{cM}$ (Table 1 and Supplementary Table 3). The numbers of SNP markers per LG, the lengths of LGs, and the length of corresponding pseudomolecules were positively correlated (Supplementary Table 3).

Similarly, the linkage map of the Jm genome constructed from the $31.09 \times \mathrm{cv}$. Serr MP consisted of 950 SNP markers, which were in 619 co-segregating marker bins, whereas that of the $J r$ genome consisted of 748 SNP markers, which were in 625 co-segregating marker bins (Table 1). The total length of the linkage map of the Jm genome was $1115.7 \mathrm{cM}$, but that for the $J r$ genome was longer, $1357.4 \mathrm{cM}$ (Table 1$)(P=0.003$, paired $t$-test). The individual LGs ranged from 50.8 to $101.0 \mathrm{cM}$ with a mean of $69.7 \mathrm{cM}$ in the $J m$ genome and from 53.3 to $132.3 \mathrm{cM}$ with a mean of $84.8 \mathrm{cM}$ in the $J r$ genome (Table 1, Supplementary Table 3$)$. In contrast to MP $31.01 \times \mathrm{cv}$. Serr, LG Jm8S was adequately covered with SNP markers (Supplementary Fig. 2). The numbers of SNP markers per LG, the genetic lengths of LGs, and the length of corresponding pseudomolecules were positively correlated (Supplementary Table 3).

\section{Resistance to Phytophthora spp}

Resistance to $P$. cinnamomi and $P$. pini was investigated in 15 separate experiments (Supplementary Tables 4, 5). Totals of 249 and 246 hybrids were tested for resistance to $P$. cinnamomi and $P$. pini, respectively, in the $31.01 \times \mathrm{cv}$. Serr population and 223 and 275 were tested for resistance to $P$. cinnamomi and $P$. pini, respectively, in the $31.09 \times$ cv. Serr population (Supplementary Table 4). From 10.2 to 11.5 clones, on average, were screened per hybrid.

Clones grown in soil inoculated with P. cinnamomi had on average 33.2 percent of crown length rotted (PCLR) whereas nearly no crown rot was observed on control plants grown in uninoculated soil (Table 2). The difference between inoculated and control plants was less pronounced when percent of root length rotted (PRLR) was employed in the quantification of resistance. Clones grown in soil inoculated with $P$. cinnamomi had on average 44.6 PRLR while plants in control soil had 22.5 PRLR (Table 2).

Clones grown in soil inoculated with $P$. pini had an average of 36.5 PCLR whereas those grown in control soil had an average of 1.2 PCLR (Table 2). However, when PRLR was measured, clones grown in soil inoculated with P. pini had an average of 36.2 PRLR and clones grown in control soil had an average of 20.5 PRLR (Table 2). The $31.09 \times \mathrm{cv}$. Serr population of hybrids, on average, had lower PCLR and PRLR means than the $31.01 \times \mathrm{cv}$. Serr population. This was true both for clones grown in control soil (for PRLR variable only) and inoculated soil (for PCLR and PRLR variables) (Table 2).

A Linear Mixed Model (LMM) was built with the PRLR and PCLR variables as described in Materials and Methods. Best Linear Unbiased Predictor (BLUP) values were exported from the fitted model and used in the QTL mapping as phenotypic data.

\section{Resistance to $A$. tumefaciens}

Response to infection with $A$. tumefaciens was analyzed in 429 hybrids and three commercial rootstocks, which were used as controls (Supplementary Table 6). Responses to inoculation were scored on a $1-4$ scale, with 1 for resistant and 4 for susceptible. A mean score in population $31.09 \times \mathrm{cv}$. Serr was slightly but significantly lower than a mean score in population $31.01 \times \mathrm{cv}$. Serr (Table 3 ).

\section{P. cinnamomi, P. pini, and A. tumefaciens resistance QTL mapping}

The BLUP PRLR and PCLR values were used in the Interval Mapping (IM) and Multi QTL Mapping (MQM) of Phytophthora spp. resistance. Both major and minor QTLs were present on the J. microcarpa genetic maps in both mapping populations (Fig. 1); none was present on the two cv. Serr maps. The major QTLs were located on 
Table 3 Mean crown gall score in populations of $J$. microcarpa 31.01 and $31.09 \times J$. regia cv. Serr hybrids

\begin{tabular}{llll}
\hline $\begin{array}{l}\text { Mapping } \\
\text { population }\end{array}$ & Hybrids (no.) & $\begin{array}{l}\text { Clones phenotyped } \\
\text { per hybrid (no.) }\end{array}$ & $\begin{array}{l}\text { Mean } \\
\text { score }^{\mathbf{a}}\end{array}$ \\
\hline $31.09 \times$ cV. Serr & 205 & 5.06 & $2.18 \mathrm{a}$ \\
$31.01 \times$ cV. Serr & 224 & 4.94 & $2.25 \mathrm{~b}$ \\
\hline
\end{tabular}

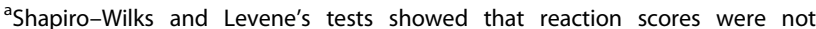
normally distributed, and variances were unequal. The non-parametric Mann-Whitney $U$ test with Bonferroni correction was used to test the significance of differences between means. Means followed by the same letter are not different at $a=0.05$
LG Jm4D (see Fig. 1 for IM and Supplementary Fig. 2 for MQM). They were statistically significant in six of the eight QTL analyses of resistance to Phytophthora spp. and explained from 12.2 to $22.2 \%$ of the variation in a MP. The logarithm of the odds (LOD) of each major QTL peaked in a similar area of LG Jm4D (Table 4 and Fig. 1). None of the minor QTLs was statistically significant (Table 4).

Responses of commercial rootstocks embedded as standards into each experiment revealed random variation among experiments (Kruskal-Wallis test, $P<0.001$ ) (Supplementary Table 5). To determine if this variation affected the QTL analysis, hybrids in each experiment

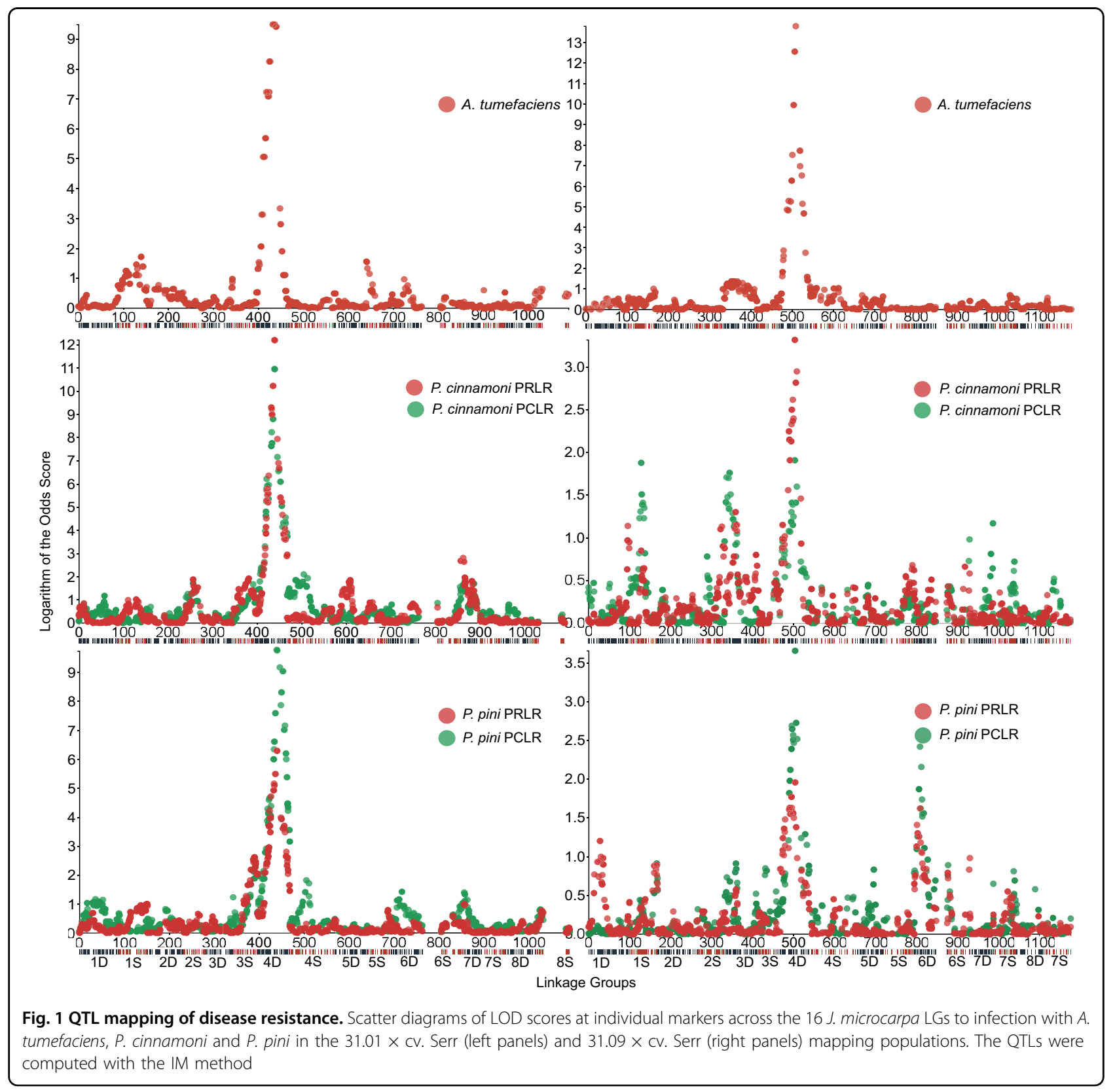


Table 4 LOD peaks of QTLs for resistance to P. cinnamomi, P. pini, and A. tumefaciens mapped with the interval mapping method

\begin{tabular}{|c|c|c|c|c|c|c|c|}
\hline Population & Pathogen & Trait & LG & LOD & Significant LOD (cM) & $\begin{array}{l}\text { LOD peak } \\
\text { location (cM) }\end{array}$ & $\%$ total variation explained \\
\hline \multirow[t]{9}{*}{$31.01 \times$ cv. Serr } & \multirow[t]{4}{*}{ P. cinn. } & \multirow[t]{2}{*}{ PCLR } & $J \mathrm{~m} 4 \mathrm{D}$ & $10.96^{\mathrm{a}}$ & $21.3-71.8$ & 43.4 & 20.2 \\
\hline & & & $\mathrm{Jm} 4 \mathrm{~S}$ & 2.09 & - & 36.8 & 4.2 \\
\hline & & \multirow[t]{2}{*}{ PRLR } & $\mathrm{Jm} 4 \mathrm{D}$ & $12.21^{a}$ & $22.6-70.3$ & $43.4-43.6$ & 22.2 \\
\hline & & & $J \mathrm{~m} 7 \mathrm{D}$ & 2.89 & - & 3.3 & 5.8 \\
\hline & \multirow[t]{4}{*}{ P. pin. } & \multirow[t]{2}{*}{ PCLR } & $\mathrm{Jm} 4 \mathrm{D}$ & $10.24^{a}$ & $21.3-71.8$ & 46.7 & 19 \\
\hline & & & $J \mathrm{~m} 3 \mathrm{~S}$ & 2.34 & - & 50.0 & 4.7 \\
\hline & & \multirow[t]{2}{*}{ PRLR } & $J \mathrm{~m} 4 \mathrm{D}$ & $6.31^{\mathrm{a}}$ & $25.9-63.2$ & 43.2 & 12.2 \\
\hline & & & $J \mathrm{~m} 3 \mathrm{~S}$ & 2.61 & - & $46.7-47.2$ & 5.2 \\
\hline & A. tum. & $C G$ & $\mathrm{Jm} 4 \mathrm{D}$ & $22.1^{\mathrm{a}}$ & $8.5-57.8$ & 37.43 & 37.7 \\
\hline \multirow[t]{4}{*}{$31.09 \times$ cv. Serr } & P. cinn. & PRLR & $J \mathrm{~m} 4 \mathrm{D}$ & $3.32^{\mathrm{a}}$ & $22.2-47.3$ & 36.7 & 7.5 \\
\hline & \multirow[t]{2}{*}{ P. pin. } & \multirow[t]{2}{*}{ PCLR } & $J \mathrm{~m} 4 \mathrm{D}$ & $3.7^{\mathrm{a}}$ & $24.4-45.3$ & 36.7 & 8.3 \\
\hline & & & Jm6D & 2.39 & $12.4-17.4$ & 13.7 & 5.5 \\
\hline & A. tum. & CG & $J \mathrm{~m} 4 \mathrm{D}$ & $17^{\mathrm{a}}$ & $8.8-66$ & 40.5 & 32.3 \\
\hline
\end{tabular}

andicate QTLs significant at $a=0.05$

were grouped into two groups based on the allele of a SNP marker closest to the LOD peak of the major QTL. Marker allele $a$ was associated with susceptibility to Phytophthora spp. and allele $b$ with resistance. In most experiments, the two populations of hybrids significantly differed from each other in at least one of the Phytophthora sp. $\times$ screening method combinations (Supplementary Fig. 3). In an experiment conducted in March 2017 and experiment 2 conducted in July 2017 none of the four Phytophthora sp. $\times$ screening method combinations yielded a significant difference between the $a$ and $b$ groups. The two experiments were removed and the QTL analyses, using the IM approach, were repeated (Supplementary Fig. 3). The LOD profiles along the genetic maps of the $J m$ genome remained similar to those when all data were used (compare Fig. 1 with Supplementary Fig. 3), indicating that variation among the experiments did not meaningfully impact the QTL analysis.

A single major QTL was also detected for resistance to $A$. tumefaciens in each MP. The A. tumefaciens resistance QTLs were in LG Jm4D, and were in a similar area where the major QTLs for resistance to Phytophthora spp. were located. The QTLs were highly significant and explained 32.3 and $37.7 \%$ of the variation in A. tumefaciens infection response in the two MPs (Table 4). There were two minor peaks of LOD $<2$, which were inconsistent between the $31.01 \times \mathrm{cv}$. Serr and $31.09 \times$ cv. Serr MPs (Fig. 1) and none was significant.

We propose here the following naming convention for Juglans QTLs. A letter Q will indicate that a QTL rather than a Mendelian locus is named. $Q$ will be followed by a three or four-letter QTL name, followed by the abbreviated name of laboratory that mapped the QTL. The name will end with the name of the chromosome on which the QTL is located. Following this convention, the three major QTLs mapped here were: QPcin.ucd.Jm4D, QPpin.ucd.Jm4D, and QAgr.ucd.Jm4D. Because the LOD peaks for QTLs based on PCLR and PRLR were co-located, it was assumed that the QTLs mapped with the two resistance screening approaches were estimates of the same QTL.

A null hypothesis that resistance to all three pathogens was associated with the same haplotype was tested by computing correlation coefficients of the resistance scores and the SNP alleles along LG Jm4D (Supplementary Table 7). The correlation coefficients should peak in the same area of the LG and all coefficients should be of the same sign if the hypothesis were true (the alphabetic allele naming used in Supplementary Table 2 was converted into numerical naming, $a=1$ and $b=2$ in Supplementary Table 7). On the $31.01 \times$ cv. Serr map, the correlation coefficients peaked between markers 31.01_m4D_26359154 and 31.01_Jm4D_26669075 for all three pathogens, and all were negative in that area, indicating that resistance to all three pathogens was associated with haplotype $b$. On the $31.09 \times \mathrm{cv}$. Serr map, the correlation coefficients peaked between markers 31.09_Jm4D_23816262 and 31.09Jm4D_26359154, and all correlation coefficients were negative indicating that resistance to all three pathogens was also associated with haplotype $b$. 
Table 5 Meta-QTL analysis of reactions to infection with P. cinnamomi, P. pini, or A. tumefaciens in linkage group Jm4D in the $J$. microcarpa $\times$ cv. Serr hybrids

\begin{tabular}{|c|c|c|c|c|c|}
\hline QTL & Population & Trait & Markers delimiting LOD peaks ${ }^{\mathrm{a}}$ & $\begin{array}{l}\text { Projection } \\
\text { on Jm4D (bp) }\end{array}$ & Mean projection of all LOD peaks (bp) \\
\hline \multirow[t]{4}{*}{ QPcin.ucd.Jm4D } & $31.01 \times$ cv. Serr & PCLR & 31.01_Jm4D_26168643 & 26168643 & 25502498 \\
\hline & & PRLR & 31.01_Jm4D_26168643 & 26168643 & \\
\hline & $31.09 \times$ cv. Serr & PRLR & 31.09_Jm4D_23816262 & 24037763 & \\
\hline & & & 31.09_Jm4D_24259264 & & \\
\hline \multirow[t]{6}{*}{ QPpin.ucd.Jm4D } & $31.01 \times$ cv. Serr & PCLR & 31.01_Jm4D_26359154 & 27006600 & \\
\hline & & & 31.01_Jm4D_27654046 & & \\
\hline & & PRLR & 31.01_Jm4D_25235336 & 25701990 & \\
\hline & & & 31.01_Jm4D_26168643 & & \\
\hline & $31.09 \times$ cv. Serr & PCLR & 31.09_Jm4D_23816262 & 24037763 & \\
\hline & & & 31.09_Jm4D_24259264 & & \\
\hline \multirow[t]{3}{*}{ QAgr.ucd.Jm4D } & $31.01 \times$ cv. Serr & CG & 31.01_Jm4D_23843516 & 24539426 & \\
\hline & & & 31.01_Jm4D_25235336 & & \\
\hline & $31.09 \times$ cv. Serr & CG & 31.09_Jm4D_26359154 & 26359154 & \\
\hline
\end{tabular}

alf a LOD peak was between markers, the two markers are listed above each other

\section{Meta-QTL analysis}

QPcin.ucd.Jm4D, QPpin.ucd.Jm4D, and QAgr.ucd.Jm4D were located in the same area of LG Jm4D and resistance to all three pathogens was conferred by the same haplotype, which suggested that resistance to the three pathogens could be conferred by a common Mendelian gene. Since the QTLs were mapped on two different maps built from different SNP markers, the eight individual QTLs could not be directly compared. To facilitate their comparison, a marker closest to each LOD peak was projected onto the Jm4D pseudomolecule ${ }^{4}$ (Table 5). If a LOD peak was between two markers, the arithmetic mean of the marker registry on the Jm4D pseudomolecule was projected. The eight Jm4D projections were used in ANOVA type III analysis to test the null hypothesis that there was no difference in the location of QPcin.ucd.Jm4D, QPpin. ucd.Jm4D, and QAgr.ucd.Jm4D. The F-test was not significant $(P=0.239)$. We, therefore, accepted the null hypothesis that there were no significant differences among the locations of QPcin.ucd.Jm4D, QPpin.ucd.Jm4D, and QAgr.ucd.Jm4D. The eight projections in bp were averaged (25,502,498 bp) (Table 5 ) and a $95 \%$ confidence interval (CI, Student $t$-distribution) was computed. The CI ranges from $24,547,096$ to $26,457,899 \mathrm{bp}$ and contains 158 high-confidence genes, from Jm4DG00123100 to Jm4DG001389004.

\section{Discussion}

In our previous studies, progeny of J. microcarpa halfsib trees 31.01 and 31.09 segregated for resistance to
Agrobacterum and Phytophthora spp. ${ }^{2,3}$, but the genetic basis was unknown. The two trees were crossed here with J. regia $\mathrm{cv}$. Serr and large populations of hybrids for mapping disease resistance were developed. The hybrids were genotyped following the GBS approach ${ }^{20}$ and four genetic maps were produced, one each for the $J m 31.01$ and Jm 31.09 genome and two for J. regia cv. Serr. The maps were deployed in QTL mapping of resistance to $A$. tumefaciens, $P$. cinnamomi, and $P$. pini.

\section{Genetic maps}

About 20\% more SNPs were obtained in the Jm genome than in the $J r$ genome in both MPs, which was consistent with greater nucleotide diversity observed in J. microcarpa $\left(\pi=8.4 \times 10^{-3}\right)$ compared to that in J. regia $(\pi=5.6 \times$ $\left.10^{-3}\right)^{19}$. Maps of the $J m$ genome were consequently built from greater numbers of markers than the cv. Serr maps. Even low genotyping and mapping error rates lead to substantial map length expansions ${ }^{23}$, which increase as the numbers of markers on such maps increase. J. microcarpa maps would be longer than the cv. Serr maps if our deployment of the GBS were burdened by errors since the J. microcarpa maps were built from larger numbers of markers than the cv. Serr maps. Both $J$. microcarpa maps were shorter than the cv. Serr maps, providing no evidence of map expansion, indicating that we were successful in keeping GBS error rates low.

The lengths of the cv. Serr maps, in addition to being longer than the J. microcarpa maps, also exceeded the lengths of the maps of $J$. regia $\mathrm{cv}$. Chandler published 
earlier $^{5,20,24}$. Chandler, like the J. microcarpa mother trees 31.01 and 31.09, was used as the female parent. Sexrelated differences in recombination rates are common in both plants and animals. Our data suggest that recombination rates are greater in the male meiosis than in the female meiosis in Juglans.

The short lengths of LGs is another notable feature of recombination in Juglans genomes 5 . On average, the genetic lengths of the Juglans chromosomes are about half of the genetic lengths typically found for chromosomes in herbs, particularly those that are self-pollinating ${ }^{5}$. Withinarm double crossovers (DCO) take place rarely in Juglans. Analyses of our four maps estimated intra-arm DCO rates to range from 4.15 to $7.26 \%$ meiotic chromosome arms (Supplementary Table 8). Juglans meiosis is thus characterized by distal crossover localization ${ }^{5,25}$ and paucity of DCO. These two tendencies produce genetically short chromosomes with distally located crossovers.

\section{Resistance QTLs}

Both J. microcarpa mother trees were heterozygous for QTLs on chromosome Jm4D conferring resistance to each of the three investigated pathogens. QTL QAgr.ucd. $J m 4 D$ explained 32.3 and $37.7 \%$ of phenotypic variation in the $31.01 \times \mathrm{cv}$. Serr and $31.09 \times \mathrm{cv}$. Serr populations, respectively. In contrast, QTLs QPcin.ucd.Jm $4 D$ and QPpin.ucd.Jm4D explained only from 8.2 to $19.3 \%$ of phenotypic variation. The lower percentage of phenotypic variation explained by the Phytophthora spp. QTLs were likely related to random variation that accompanied the PCLR and PRLR assays. Another source of variation could have been individual screening "experiments". Nearly 20,000 clones were screened for resistance to Phytophthora spp., which necessitated screening for resistance to Phytophthora spp. over a period of four years in 15 different times (experiments). To reduce random variation due to this source, clones of three rootstocks were embedded as standards in each experiment, a mixed linear model was built, and BLUP values were computed and used in QTL mapping as phenotypic data. Our posteriori analysis of the QTL data revealed that this approach minimized the experiment effect on the results of the QTL analysis.

The LOD score for the QAgr.ucd.Jm4D, QPcin.ucd. $J m 4 D$, and QPpin.ucd.Jm4D QTLs peaked in the same general area of LG Jm4D and resistance scores for all three pathogens correlated with haplotype $b$ in both MPs. We, therefore, hypothesized that the resistance to all three pathogens had a common genetic cause. Meta-QTL analysis placed with $95 \%$ probability this hypothetical locus into an interval $24,547,096-26,457,899 \mathrm{bp}$ on pseudomolecule Jm4D. This interval contains 158 high-confidence genes, from Jm4DG00123100 to Jm4DG00138900.

It could be that the co-location of the three QTLs is just a coincidence caused by high density of resistance genes in that region of the Jm4D chromosome. This region of the $J m$ genome has been shown to harbor one of the highest concentrations of resistance genes and some are in the Jm4DG00123100 to Jm4DG00138900 interval ${ }^{4}$. Alternatively, the co-location of resistance to these different pathogens could be due to broad spectrum resistance (BSR) conferred by a single locus ${ }^{26}$. There are numerous examples in which resistance to multiple diseases has appeared to be co-located on a map, but unequivocal evidence for BSR requires isolation of the resistance gene, which we have not done, and cannot decide between these two alternatives.

In an attempt to find a candidate gene for Phytophthora spp. resistance, the QTL for resistance to $P$. cinnamomi on Jm4D was compared to QTLs for resistance to $P$. cinnamomi mapped in Japanese and Chinese chestnuts. In both chestnut species, resistance to $P$. cinnamomi was mapped in LGs $\mathrm{E}$ and $\mathrm{K}^{15,16}$ indicating conservation of the two QTLs in Castanea. We hypothesized that if the region on Jm4D harboring the major QTL is homoeologous to any of the two Castanea regions, shared genes could suggest candidate resistance genes. Homology was examined for 247 genes located in the region from 24 to $27 \mathrm{Mb}$ on the Jm4D pseudomolecule against scaffold sequences of Chinese chestnut https://www.hardwoodgenomics.org/ Genome-assembly/1962958 at the QTLs on LGs E and K. Only one of the 247 genes, a resistance analogue Jm4DG00140500, was shared indicating J. microcarpa and Castanea QTLs were not orthologous.

\section{Rootstock breeding}

Crown gall and Phytophthora root and crown rots are devastating soil-borne diseases of commercial walnut orchards in California. Currently, California rootstocks have some resistance to single pathogens ${ }^{2,3}$, but none is known to be highly resistant to two or more pathogens. A remarkable feature of resistance segregating in the progeny of $J$. microcarpa trees 31.01 and 31.09 is that resistance to A. tumefaciens and both Phytophthora spp. is mediated by the same region of chromosome Jm4D and by the same haplotype. These attributes and availability of SNP markers for selection of this resistance (Supplementary Table 1) will greatly simplify stacking resistance to both A. tumefaciens and Phytophthora spp. in a single rootstock.

\section{Materials and methods \\ Plant materials}

Juglans microcarpa trees DJUG 31.01 and DJUG 31.09 (USDA National Clonal Germplasm Repository, Davis, https://www.ars.usda.gov/pacific-west-area/davis-ca/natlclonal-germplasm-rep-tree-fruit-nut-crops-grapes/) were used as mother trees in hybridization with J. regia cv. Serr in the springs of 2012-2015. Female flowers were sealed 


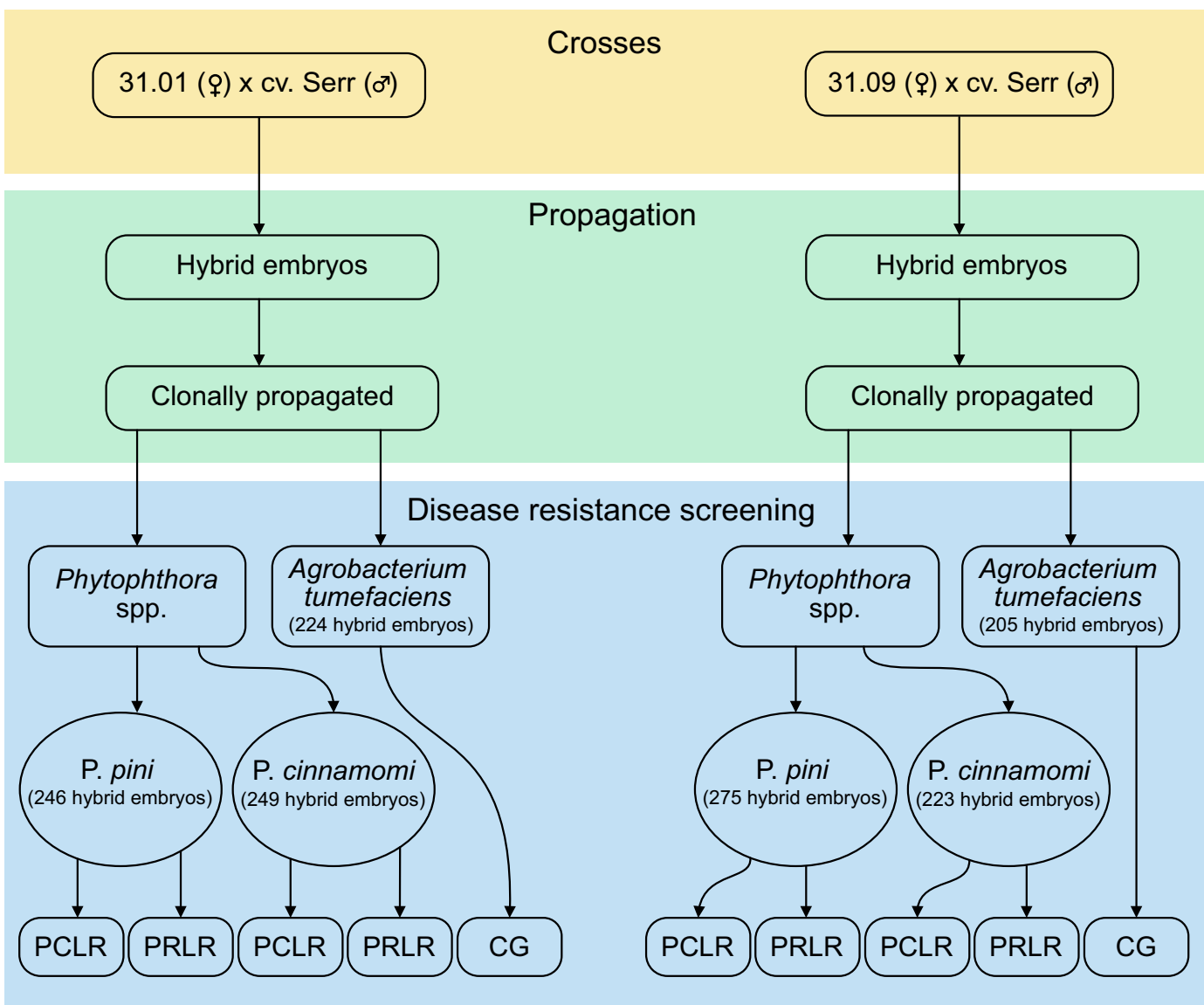

Fig. 2 Production, clonal propagation, and disease screening of J. microcarpa $\times$ J. regia hybrids. The bottom row indicates trait abbreviations: percent crown length rotted (PCLR), percent root length rotted (PRLR), and crown gall resistance score (CG)

in pollen-impermeable semi-porous bags (PBS 10-1, PBS International) prior to opening, using non-absorbent cotton to cushion and seal the bags around the branch after male flowers had been removed. When female flowers began opening (at the stigma separation stage), previously collected cv. Serr pollen was injected into the bags using a syringe. The bags were removed 3-4 weeks after pollination and immature nuts were tagged. The nuts were collected while still immature and with intact hulls (July-August), and stored refrigerated (up to several weeks). Embryos were extracted from nuts, germinated in vitro, and micropropagated ${ }^{27}$ to produce clones for disease resistance testing.

\section{Resistance to $P$. cinnamomi and P. pini}

Rooted clonal plants derived from $31.01 \times \mathrm{cv}$. Serr and $31.09 \times \mathrm{cv}$. Serr hybrids were transferred to $0.5-\mathrm{L}$ pots containing UC potting $\mathrm{mix}^{28}$ and grown in a greenhouse. When the plants reached at least $15 \mathrm{~cm}$ in height, they were entered into greenhouse evaluations of resistance to Phytophthora spp. The numbers of hybrid progeny evaluated for resistance to $P$. cinnamomi and P. pini (formerly
P. citricola sensu lato) across 15 experiments are specified in Fig. 2. Each experiment included three clonal rootstocks with known levels of resistance as standards: 'AX1' (low resistance to both $P$. cinnamomi and P. pini), J. microcarpa $\times J$. regia ' $\mathrm{RX1}$ ' (highly resistant to $P$. cinnamomi and moderately resistant to $P$. pini), and wingnut (Pterocarya sp) 'WNxW' (highly resistant to both P. cinnamomi and P. pini). Evaluations of resistance to Phytophthora spp. were avoided during fall and winter (November-January), because it was determined that susceptibility to Phytophthora spp. tends to be reduced during that period.

Four isolates of P. cinnamomi (GB647, GB5972, GB5973, and GB6378) were combined for inoculation. Likewise, four isolates of P. pini (GB572, GB5181, GB5781a, and GB5997k) were combined for inoculation. Plants were inoculated either with $P$. cinnamomi or $P$. pini. All of the isolates were from walnut trees affected by Phytophthora root and crown rot in the Central Valley of California and had been hyphal tipped. Identity of the isolates was confirmed by sequencing the rRNA gene internal transcribed spacer (ITS) ${ }^{2}$. All of the isolates 
possessed morphological traits (e.g., sporangia shape and papilla type, hyphal morphology, and the presence and morphology of oogonia and antheridia) characteristic of their identity. The combination of four isolates was used for each Phytophthora sp. to ensure that resistance determined in the greenhouse would also be expressed in California orchards. There is no evidence for the existence of Phytophthora races in California walnut orchards or walnut cultivar $\times$ Phytophthora isolate interactions for either P. cinnamomi or P. pini. The use of multiple Phytophthora isolates to represent a Phytophthora sp. is a common practice in Phytophthora sp. resistance QTL studies in woody perennials, e.g. ${ }^{16}$. Similarly, QTLs for resistance to $P$. cactorum in strawberry were mapped using multiple-isolate mixtures ${ }^{29}$.

Inoculum was prepared, plants were inoculated, and an experimental design previously described ${ }^{2}$ for phase 2 screening was used. Two months after inoculation, PCLR and PRLR were assessed as described previously ${ }^{2}$. Also, after the disease assessments, culture-based isolations were used as described previously ${ }^{2}$ to determine that plants grown in non-infested soil had remained free from Phytophthora and that those grown in soil infested with $P$. cinnamomi or P. pini had been exposed to the appropriate pathogen.

In statistical analysis, PCLR and PRLR values from Phytophthora sp.-inoculated plants were compared to those from control plants using the Mann-Whitney $U$ test. Resistance among hybrids was evaluated across multiple greenhouse experiments. A Kruskal-Wallis test was used to assess the effect of experiment on phenotypes. Linear Mixed Model (LMM) implemented in lme4 v1.1.18.1 $\mathrm{R}$ package ${ }^{30}$ was used to assess the effects of random variables on the response variable. Genotype and experiment were random variables in the LMM model, while the disease phenotype scores were response variables. Normality of fitted model residuals was visualized using a histogram and tested using Shapiro-Wilk test. BLUP values were extracted from the model and used in-place of phenotype values in the QTL analysis.

\section{Resistance to $A$. tumefaciens}

Rooted clonal plants derived from $31.01 \times \mathrm{cv}$. Serr and $31.09 \times \mathrm{cv}$. Serr hybrids were transferred to $0.5-\mathrm{L}$ pots containing UC potting mix and cultivated in the greenhouse until the stems were at least $0.5 \mathrm{~cm}$ in diameter. Three to six clonal plants per hybrid were used for $A$. tumefaciens resistance screening. Commercial walnut rootstocks VX211, Vlach (J. hindsii $\times$ J. regia), and RX1 were used as standards. Groups of nine unique clones, i.e., each derived from a different hybrid or check, were randomly selected and placed into plastic bins. Bins were randomized on a greenhouse bench.
Colonies of A. tumefaciens strain EC1 were grown for $48 \mathrm{~h}$ at $28^{\circ} \mathrm{C}$ on tryptic soy agar (TSA). Ten $\mathrm{mL}$ of tryptic soy broth (TSB) was inoculated with a single colony and grown overnight on a shaker at $210 \mathrm{rpm}$ and $28^{\circ} \mathrm{C}$. The TSB culture was pelleted in a Sorvall RC 5C Plus centrifuge (Fisher Scientific, Waltham, MA) at $4575 \mathrm{~g}$ in a SH3000 swinging bucket rotor at room temperature. The bacterial cell pellet was washed twice with sterile water. The pellet was suspended in sterile water and adjusted to absorbance $600_{\mathrm{nm}}=1.0\left(\sim 10^{9} \mathrm{CFU} / \mathrm{ml}\right)$ using a Beckman DU 800 spectrophotometer (Beckman, Fullerton, CA).

Two joined $0.9 \mathrm{~cm}$ metal Exacto blades (ACE hardware, Oak Brook, IL) were dipped into $2 \mathrm{~mL}$ of an A. tumefaciens suspension, and the A. tumefaciens infested blades were pierced $2 \mathrm{~mm}$ deep into the main stem at two positions approximately $0.5 \mathrm{~cm}$ above the soil line. The blade was dipped again into the suspension and a second stab wound was made on the stem, $2.5 \mathrm{~cm}$ above the first stab wound. The resulting vertical stabs were wrapped with parafilm. A single clone per hybrid was stabbed with a blade dipped in sterile water, which served as a negative control. Susceptible hosts, Datura stramonium and Solanum lycopersicum (tomato), were inoculated with the same A. tumefaciens EC1 suspension, which served as positive controls. Plants were grown in the greenhouse with natural light supplemented with $16 \mathrm{~h}$ of overhead LED lighting daily and temperature $10-22^{\circ} \mathrm{C}$ during the day and $10-17^{\circ} \mathrm{C}$ at night.

Plants were examined for gall development two months after inoculation. The following numerical system was used to record the percentage of stem girdling by gall tissue: $1=$ no gall or tumor, $2=<25 \%$ of stem girdled, $3=25-50 \%$ stem girdled, and $4=>50 \%$ stem girdled. A rating of 1 indicated a highly resistant/tolerant response. A rating of 2 indicated a partially resistant response, and 3 and 4 indicated susceptible and highly susceptible response, respectively.

\section{GBS and SNP discovery}

Genomic DNA was isolated with the CTAB plant genomic DNA isolation method ${ }^{31}$ from micro-propagated $\mathrm{F}_{1}$ hybrids and J. microcarpa and cv. Serr parents. DNA was diluted to the uniform concentration of $55 \mathrm{ng} / \mu \mathrm{l}$. GBS involved complexity reduction by $P s t I$ restriction enzyme digestion ${ }^{21}$. Groups of 96 samples including both parents were multiplexed using unique barcodes, and single-end sequenced with the Illumina HiSeq2000 platform at the Genomic Diversity Facility of Institute of Biotechnology, Cornell University. SNPs were called with Tassel-GBS pipeline v2.0 $0^{32}$ using default settings except that a minimum mapping quality of 2 was used in SAMToGBSdbPlugin. Illumina reads were deconvoluted using the barcodes, reads were filtered and trimmed to $64 \mathrm{bp}$ to construct unique tags. The $J$. microcarpa Jm31.01_v1.0 and J. regia JrSerr_v1.0 genome sequences ${ }^{4}$ were combined 
into a single fasta file and used as a reference. Unique tags were aligned onto the reference sequences with the BWAaln aligner. High-quality SNPs were selected among SNPs obtained from the Tassel-GBS pipeline with VCFtools ${ }^{33}$, BCFtools $^{34}$, and in-house awk and Python scripts. Nonsegregating SNPs and calls below a threshold of five reads were removed.

The following convention was implemented in naming the markers. Each name was started with that of the mapping population followed by the abbreviated names of the genome and chromosome, and ended with the location of the SNP in the registry of the corresponding JrSerr_v1.0 or Jm31.01_v1.0 pseudomolecule ${ }^{4}$.

To verify that pollen contamination or some other perturbation had not occurred during the development of hybrids, the SNP data were analyzed with a Principal Component Analysis (PCA) implemented in the Tassel v5.022.

\section{Linkage map construction}

Linkage maps were constructed separately for the $J$. microcarpa and cv. Serr genomes in each mapping population using the Joinmap v4.1 mapping program $^{35}$. SNP markers showing segregation distortion $\left(P<0.001, \chi^{2}\right.$ test) were removed. Remaining markers were grouped into linkage groups (LGs) by a regression mapping procedure $^{36}$ with a minimum threshold logarithm of odds (LOD) score of 8 and a maximum recombination fraction of 0.3 . The Joinmap v4.1 $1^{35}$ assigned sequential numbers to the loci after computing the groupings of co-segregating markers. For a bin of co-segregating markers, the first marker in a bin was chosen as the representative marker of the bin by the mapping program. Recombination fractions were converted into map distances $(\mathrm{cM})$ with the Kosambi mapping function ${ }^{37}$. Marker order along each LG was confirmed by comparing it with the order along the reference genome pseudomolecule.

\section{QTL analysis}

QTLs were mapped separately in each mapping population with MapQTL v6.0 ${ }^{38}$. IM with one cM increments was employed to locate putative QTLs. QTLs with significant LOD threshold $(\alpha=0.05)$ values were identified with a permutation test with 1000 independent permutations. SNPs exceeding significant threshold LOD $(\alpha=$ $0.05)$ were used as initial cofactors (https://www.kyazma. $\mathrm{nl} /$ docs/MQ6Manual.pdf). Cofactors that were significant were identified with the automatic cofactor identification method and used in $\mathrm{MQM}^{39,40}$. Percentage of variance explained by each QTL and their additive genetic effects were extracted from MQM.

\section{Statistical analysis and data visualization}

Statistical tests were performed using either $\mathrm{R}^{41}$ or Python SciPy v1.0 library ${ }^{42}$. Linkage maps were drawn using our in-house JavaScript library built on top of $\mathrm{d} 3 \mathrm{js}^{43}$. Bar graphs and histograms were drawn using $\mathrm{R}$, Matplotlib ${ }^{44}$, or Seaborn Python (http://seaborn.pydata. org) packages.

\section{Acknowledgements}

This work was supported in part by USDA NIFA-SCRI grants no. 59-5306-2-333 and 2018-51181-28437, the California Walnut Board, USDA ARS Project: 203222000-016-00D, and NIFA-Hatch 1010599. We also thank Steven Lee, Kristina McCreery, llean Tracy, Sierra Gold Nurseries, and Duarte Nursery for their invaluable help in the clonal propagation of all members of both walnut mapping populations.

\section{Author details}

${ }^{1}$ Department of Plant Sciences, University of California, Davis, USA. ${ }^{2}$ USDA-ARS Crops Pathology and Genetics Research Unit, Department of Plant Pathology, University of California, Davis, USA. ${ }^{3}$ National Clonal Germplasm Repository, USDA-ARS, University of California, Davis, USA. ${ }^{4}$ Department of Nematology, University of California, Riverside, USA

\section{Author contributions}

D.A.K. and A.W. organized and lead efforts to acquire extramural funding. M.-C. L., D.V., M.A., P.J.B., R.K.R., and A.M.D. generated and analysed the GBS data and performed mapping. R.K.R. and J.D. generated the first draft of the paper. G.T.B., D.A.K., A.W., A.M., and N.O. led the disease resistance screening efforts. C.A.L. led the in vitro clonal propagation. All authors read, edited, and approved the final draft.

\section{Data availability}

All data generated in this study are included in this published article and its supplementary information.

\section{Conflict of interest}

The authors declare no competing interests.

Supplementary information The online version contains supplementary material available at https://doi.org/10.1038/s41438-021-00546-7.

Received: 14 September 2020 Revised: 16 February 2021 Accepted: 26 March 2021

Published online: 01 May 2021

\section{References}

1. Potter, D., Gao, F. Y., Baggett, S., McKenna, J. R. \& McGranahan, G. H. Defining the sources of Paradox: DNA sequence markers for North American walnut (Juglans L.) species and hybrids. Sci. Hortic.-Amst. 94, 157-170 (2002).

2. Browne, G. T. et al. Resistance to species of Phytophthora identified among clones of Juglans microcarpa $\times$ J. regia. Hortscience 50, 1136-1142 (2015).

3. Kluepfel, D. A. et al. Evaluation of wild walnut Juglans spp. for resistance to crown gall disease. Phytopathology 101, S92-S92 (2011).

4. Zhu, T. et al. Sequencing a Juglans regia $\times J$. microcarpa hybrid yields highquality genome assemblies of parental species. Hort. Res. 6, 55 (2019).

5. Luo, M.-C. et al. Synteny analysis in Rosids with a walnut physical map reveals slow genome evolution in long-lived woody perennials. BMC Genomics 16, https://doi.org/10.1186/S12864-015-1906-5 (2015).

6. Sule, S. \& Burr, T. J. The effect of resistance of rootstocks to crown gall (Agrobacterium spp.) on the susceptibility of scions in grape vine cultivars. Plant Pathol. 47, 84-88 (1998).

7. Kuczmog, A. et al. Mapping of crown gall resistance locus Rcg1 in grapevine. Theor. Appl. Genet. 125, 1565-1574 (2012).

8. Bliss, F. A., Almehdi, A. A., Dandekar, A. M., Schuerman, P. L. \& Bellaloui, N. Crown gall resistance in accessions of 20 Prunus species. Hortscience $\mathbf{3 4}$, 326-330 (1999).

9. Moriya, S. et al. Evaluation and inheritance of crown gall resistance in apple rootstocks. J. Jpn. Soc. Hort. Sci. 77, 236-241 (2008).

10. Moriya, S. et al. Genetic mapping of the crown gall resistance gene of the wild apple Malus sieboldii. Tree Genet. Genomes 6, 195-203 (2010). 
11. Zhou, L. et al. Varietal differences in resistance to crown gall disease among rose cultivars by in vitro testing. J. Jpn. Soc. Hort. Sci. $\mathbf{6 8}, 440-445$ (1999).

12. Robbs, S. L., Hawes, M. C., Lin, H. J., Pueppke, S. G. \& Smith, L. Y. Inheritance of resistance to crown gall in Pisum sativum. Plant Physiol. 95, 52-57 (1991).

13. Nam, J., Matthysse, A. G. \& Gelvin, S. B. Differences in susceptibility of Arabidopsis ecotypes to crown gall disease may result from a deficiency in T-DNA integration. Plant Cell 9, 317-333 (1997).

14. Mysore, K. S., Nam, J. \& Gelvin, S. B. An Arabidopsis histone H2A mutant is deficient in Agrobacterium T-DNA integration. Proc. Natl Acad. Sci. USA 97, 948-953 (2000).

15. Santos, $C$. et al. First interspecific genetic linkage map for Castanea sativa $x$ Castanea crenata revealed QTLS for resistance to Phytophthora cinnamomi. Plos ONE 12, https://doi.org/10.1371/journal.pone.0184381 (2017).

16. Zhebentyayeva, T. N. et al. Dissecting resistance to Phytophthora cinnamomi in interspecific hybrid chestnut crosses using sequence-based genotyping and QTL mapping. Phytopathology 109, 1594-1604 (2019).

17. Paterson, A. H. et al. Resolution of quantitative traits into Mendelian factors by using a complete linkage map of restriction fragment polymorphism. Nature 225, 721-726 (1988).

18. You, F. M. et al. Genome-wide SNP discovery in walnut with an AGSNP pipeline updated for SNP discovery in allogamous organisms. BMC Genomics 13, 354 (2012).

19. Stevens, K. A. et al. Genomic variation among and within six Juglans species. G3: Genes|Genomes|Genetics, https://doi.org/10.1534/g3.118.200030 (2018).

20. Marrano, A., Sideli, G. M., Leslie, C. A., Cheng, H. \& Neale, D. B. Deciphering of the genetic control of phenology, yield, and pellicle color in Persian walnut (Juglans regia L.). Front Plant Sci. 10, 1140 (2019).

21. Elshire, R. J. et al. A robust, simple genotyping-by-sequencing (GBS) approach for high diversity species. Plos ONE 6, e19379 (2011).

22. Bradbury, P. J. et al. TASSEL: software for association mapping of complex traits in diverse samples. Bioinformatics 23, 2633-2635 (2007).

23. Lincoln, S. E. \& Lander, E. S. Systematic detection of errors in genetic linkage data. Genomics 14, 604-610 (1992).

24. Aradhya, M. K. et al. A fine-scale genetic linkage map reveals genomic regions associated with economic traits in walnut (Juglans regia). Plant Breed. 138, 635-646 (2019).

25. Marrano, A. et al. High-quality chromosome-scale assembly of the walnut (Juglans regia L.) reference genome. Gigascience 9, https://doi.org/10.1093/ gigascience/giaa050 (2020)
26. Wisser, R. J., Sun, Q., Hulbert, S. H., Kresovich, S. \& Nelson, R. J. Identification and characterization of regions of the rice genome associated with broad-spectrum, quantitative disease resistance. Genetics 169, 2277-2293 (2005).

27. Leslie, C. \& McGranahan, G. H. In Biotechnology in Agriculture and Forestry, High-Tech and Micropropagation II Vol. 18 (ed Y. P. S. Bajaj) 136-150 (SpringerVerlag, 1992).

28. Matkin, O. P., Chandler, P. A. \& Baker, K. F. In UC system for producing healthy container-grown plants (ed K. F. Baker) 68-85 (University of California, Berkeley, CA, 1957).

29. Mangandi, J. et al. pedigree-based analysis in a multiparental population of octoploid strawberry reveals QTL alleles conferring resistance to Phytophthora cactorum. G3-Genes Genom. Genet. 7, 1707-1719 (2017).

30. Bates, D., Machler, M., Bolker, B. M. \& Walker, S. C. Fitting linear mixed-effects models using Ime4. J. Stat. Softw. 67, 1-48 (2015).

31. Murray, M. G. \& Thompson, W. F. Rapid isolation of high molecular weight plant DNA. Nucleic Acids Res. 8, 4321-4325 (1980).

32. Glaubitz, J. C. et al. TASSEL-GBS: a high capacity genotyping by sequencing analysis pipeline. Plos ONE 9, https:/doi.org/10.1371/journal.pone.0090346 (2014).

33. Danecek, P. et al. The variant call format and VCFtools. Bioinformatics 27, 2156-2158 (2011).

34. Danecek, P. \& McCarthy, S. A. BCFtools/csq: haplotype-aware variant consequences. Bioinformatics 33, 2037-2039 (2017).

35. JoinMap ${ }^{\circledR}$ 4.1. Software for calculation of genetic linkage maps in experimental populations (Kyazma B. V., Wageningen, the Netherlands, 2006).

36. Stam, B. Construction of integrated linkage maps by means of a new computer package: JoinMap. Plant J. 5, 739-744 (1993).

37. Kosambi, D. D. The estimation of map distances from recombination values. Ann. Eugen. 12, 172-175 (1943).

38. MapQTL ${ }^{\circledR}$ 6, Software for the mapping of quantitative trait in experiment populations of diploid species (Kyazma B. V., Wageningen, Netherlands, 2009).

39. Jansen, R. C. Interval mapping of multiple quantitative trait loci. Genetics $\mathbf{1 3 5}$ 205-211 (1993).

40. Jansen, R. C. Controlling the type I and type II errors in mapping quantitative trait loci. Genetics 138, 871-881 (1994).

41. R: A language and environment for statistical computing (https://www.Rproject.org/, Foundation for Statistical Computing, Vienna, Austria. URL, 2018).

42. Virtanen, P. et al. SciPy 1.0: fundamental algorithms for scientific computing in Python. Nat. Methods 17, 261-272 (2020).

43. D3. js-data-driven documents (2016).

44. Hunter, J. D. Matplotlib: a 2D graphics environment. Comput. Sci. Eng. 9, 90-95 (2007). 